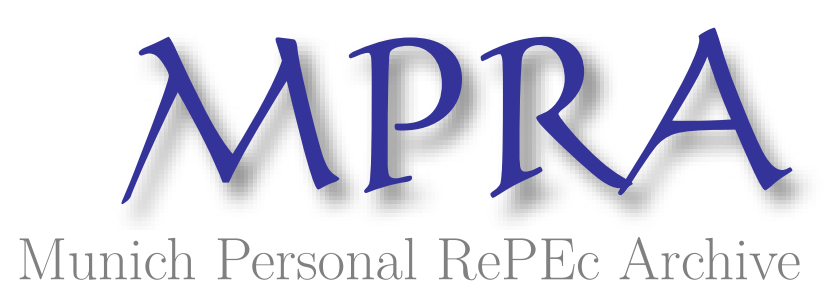

\title{
Seller Competition by Mechanism Design
}

Damianov, Damian

University of Texas - Pan American

23 June 2008

Online at https://mpra.ub.uni-muenchen.de/9348/

MPRA Paper No. 9348, posted 28 Jun 2008 04:07 UTC 


\title{
Seller Competition by Mechanism Design*
}

\author{
Damian S. Damianov ${ }^{\dagger}$ \\ University of Texas-Pan American
}

June 23, 2008

\begin{abstract}
In the market game presented here, sellers offer trade mechanisms to buyers, and buyers randomize over the sellers they visit. The distribution of buyers across sellers is endogenous and depends on all of the transaction opportunities existing in the market. Sellers choose from a broad class of trade mechanisms; the only constraints imposed on mechanisms is that they are direct, incentive compatible, and anonymous. In the (subgame perfect) equilibrium of this market, sellers hold auctions with an efficient reserve price but charge an entry fee. The entry fee depends on the number of buyers and sellers, the distribution of buyer valuations, and the buyer cost of entering the market. As the size of the market increases, the entry fee decreases and vanishes in the limit. The model sheds light on the endogenous formation of trading institutions in decentralized markets.
\end{abstract}

Key Words: competition, mechanism design, auctions

JEL Classification: D44, D82.

${ }^{*}$ This paper is a substantially revised version of Chapter 6 of my $\mathrm{PhD}$ thesis at the University of Heidelberg. I thank Jürgen Eichberger and Jörg Oechssler for their guidance and support. I am grateful for the very helpful suggestions and comments from Subir Bose, Roberto Burguet, Indranil Chakraborty, George Deltas, Chaim Fershtman, Ed Green, Hans Haller, Georgia Kosmopoulou, Vijay Krishna, Preston McAfee, Mike Peters, Angel Hernando Veciana, Tim Worrall, and from seminar participants at the 2005 Winter Meeting of the Econometric Society in Istanbul, University of Heidelberg, Keele University, Penn State University, and University of Texas-Pan American. Any remaining errors are mine.

${ }^{\dagger}$ Department of Economics and Finance, University of Texas-Pan American, 1201 West University Drive, Edinburg, Texas 78539, USA, email: ddamianov@utpa.edu 


\section{Introduction}

This paper presents a decentralized model of trade in which sellers compete for a common pool of customers by way of designing trade mechanisms. This type of competition arises in a variety of decentralized markets, e.g. markets for real estate, rental housing, used cars, and goods traded via the Internet. The model analyzes the endogenous formation of trading institutions in a setting where sellers offer trade mechanisms to buyers, and buyers decide which seller to go to. The distribution of buyers across sellers depends on all the trade mechanisms existing in the market. Sellers are free to use essentially any transaction mechanism; the only constraints imposed on mechanisms is that they are direct, incentive compatible, and anonymous.

The main purpose of this paper is to describe the trade mechanisms that sellers will use in the (subgame perfect) equilibrium of this decentralized trading game. I explore how the structure of the transaction mechanisms shapes the distribution process of buyers across sellers, and I formulate the mechanism design problem of a seller who competes with other sellers. Although this model paints a highly stylized picture of trade, and abstracts from many of the institutional details of each of the aforementioned markets, it helps gain valuable insights into the nature of competition by mechanism design and the distribution of rents in such decentralized trading environments. By obtaining an explicit solution for the equilibrium trade mechanism, the analysis provides a number of comparative statics results, and states testable implications that are not available in the standard monopoly framework.

In the symmetric (subgame perfect) equilibrium of this model sellers hold auctions. These auctions have a trivial reserve price (equal to sellers' use value of the good) and involve an entry fee. The equilibrium entry fee depends on the number of buyers and sellers, the distribution of buyer valuations, and the buyer cost of entering the market. The departure from the monopoly paradigm changes markedly the results from the received literature on auctions with entry (see e.g. Levin and Smith 1994). It raises a variety of new questions and yields a number of new insights. First, in contrast to the monopoly formulation of the model, sellers in general do not expropriate the entire surplus from buyers. Second and perhaps most surprising finding is that in some circumstances the availability of one additional seller can raise rather than diminish the entry fees used by sellers in equilibrium. Finally, when the number of buyers and sellers increases, but their ratio remains the same (i.e. the thickness of the market increases), the entry fee decreases, 
and in the limit converges to zero. The competition among sellers intensifies with the size of the market, and in large markets sellers receive a lower surplus compared to buyers. For instance, in a market with 2 buyers and 2 sellers only, buyers receive on average $40 \%$ of the total surplus generated by trade. In contrast, in a market populated by 1000 buyers and 1000 sellers, buyers receive $71.8 \%$ of the trade surplus.

The existing literature on competition in trade mechanisms has taken two distinct modelling approaches. The one strand of this literature considers markets with infinitely many buyers and sellers. Appealing to large markets, McAfee (1993), Peters (1997), and Peters and Severinov (1997) propose concepts of a market equilibrium that combine elements of game-theoretical and competitive-equilibrium analysis. These equilibrium notions require, that if a seller deviates to another mechanism, there will be no consequences for the expected payoff of bidders who visit other sellers. This approach allows the formulation of tractable frameworks within which the discussion of equilibrium trade mechanisms is possible. Yet, it remains unclear whether results based on these competitive notions of equilibrium have any bearing on markets with a small number of buyers and sellers, or even how they can be applied to markets with a large but finite number of agents (see McAfee 1993, p.1303). The approach presented here, in contrast, will be genuinely gametheoretical and will capture all the repercussions that one seller's transaction mechanism has on the payoffs of other market participants. The paper enriches this literature in three respects. First, it provides an explicit solution for the equilibrium mechanisms of markets of any size, thus covering the gap between the monopoly and the perfectly competitive case. Second, it reconfirms the findings by McAfee (1993) and Peters (1997) by demonstrating that in the limit, when the number of buyers and sellers converges to infinity, the equilibrium trade mechanisms indeed converge to an auction with a trivial reserve price and a zero entry fee. This limit is taken with respect to the subgame perfect equilibria rather than obtained at the cost of assuming away strategic effects. Finally, the model allows a comparative statics analysis with respect to the number of buyers and sellers that cannot be conducted when the number of agents is infinite.

The other strand of this literature (e.g., Hernando-Veciana 2005, Burguet and Sakovics 1999, Moldovanu, Sella, and Shi 2008) models competition among sellers who choose reserve prices in second-price auctions. ${ }^{1}$ This assumption creates tractable settings by allowing a seller's transaction mechanism to be described by a single variable. Here, in contrast, the

\footnotetext{
${ }^{1}$ The model by Moldovanu, Sella, and Shi (2008) is a multi-unit auction model in which sellers use uniform-price auctions.
} 
use of auctions will be a derived result rather than an assumption, and, as we will see, sellers prefer to use entry fees rather than reserve prices.

This paper is also related to the body of literature that endogenizes the bidder entry process into a single auction (see McAfee and McMillan 1987b, Engelbrecht-Wiggans 1993, Levin and Smith 1994, Chakraborty and Kosmopoulou 2001). Entry is associated with a fixed cost and proceeds until the expected gain from participating in the auction is entirely dissipated (i.e. is equal to the cost of entering the market). When bidders have independent private values, the seller optimally sets a trivial reserve price (equal to his use value of the good), and possibly a positive entry fee that fully expropriates buyer surplus. ${ }^{2}$ This paper follows a similar modelling approach, but accommodates any number of buyers and sellers. The availability of multiple sellers has significant implications. Interestingly, and somewhat counter-intuitively, there are circumstances in which a higher number of sellers leads to higher entry fees. The availability of more sellers means more transaction opportunities to buyers, and as a consequence, more buyers are willing to incur the cost of entering the market. In some circumstances an individual seller who raises his entry fee will see a smaller outflow of buyers when there are other sellers present in the market. This effect occurs because buyers distribute across the higher number of sellers rather than leave the market. Yet, in contrast to the literature on monopoly, in the imperfect competition case sellers do not always expropriate the entire surplus from buyers.

The rest of the paper is organized as follows. Section 2 describes the market game and Section 3 offers an overview of the results. The main statements and theorems are collected in Section 4. Section 5 presents comparative statics results and Section 6 concludes.

\section{The model}

There is a finite number of sellers indexed by $j \in J$ and a finite number of buyers indexed by $i \in I$, and I will use $J(I)$ to denote both the set and number of sellers (buyers). Each seller has one unit for sale of a homogeneous good, and each buyer seeks to buy one unit. All agents are risk neutral. The use value of the good is the same for each seller, and without loss of generality is normalized to zero. Sellers compete by simultaneously offering trade mechanisms to buyers. Each buyer decides whether to enter the market, and which seller to go to. Entry into the market is associated with fixed costs of $c \geq 0$. These costs

\footnotetext{
${ }^{2}$ The entry fee, however, is not raised beyond the point which causes bidders to stay out of the market with positive probability.
} 
might, for example, be either transportation costs or the costs a bidder incurs to inspect the good for sale and learn his valuation. These might also be the costs associated with the preparation and submission of a bid. Upon visiting a seller, each bidder learns his valuation and submits a bid. ${ }^{3}$ Every buyer has a privately observed valuation, which is an independent draw from a distribution with support normalized to $[0,1]$ and a continuously differentiable distribution function $F$. A mechanism prescribes an allocation and a payment for any number (and identity) of bidders and any realization of their valuations. ${ }^{4}$ Let us denote the set of the subsets (the power set) of all bidders by $\mathcal{I}$ and the power set of all rivals of bidder $i$ by $\mathcal{I}^{-i}$. Let $s \in \mathcal{I}$ denote a group of bidders and let $x^{s}$ be the ordered vector $^{5}$ of their valuations. Further, let $X^{s}$ denote the set of all possible ordered vectors of valuations of the bidders from group $s$. We denote by $X \equiv \bigcup_{s \in \mathcal{I}} X^{s}$ the set of all ordered vectors of the valuations of all subsets of bidders and by $x$ an element of this set ${ }^{6}$. Similarly, $X_{-i}$ denotes the set of ordered vectors of the valuations of all subsets of bidders that do not contain bidder $i$.

\subsection{Seller strategy space (trade mechanisms)}

A mechanism consists of an allocation rule, $p_{i}: X \rightarrow[0,1]$, and a payment rule, $z_{i}: X \rightarrow \mathbb{R}$. The allocation rule maps bidders' messages (bids) into a probability of winning for each bidder $i \in I$, and the payment rule maps bidders' messages into an expected payment of each bidder $i$ to the seller. I denote the strategy set of sellers by

$$
\mathfrak{A} \equiv\left\{\left\{\left(p_{i}, z_{i}\right)\right\}_{i \in I} \mid\left(p_{i}, z_{i}\right): X \rightarrow[0,1] \times \mathbb{R}\right\}
$$

\footnotetext{
${ }^{3}$ Following the modelling approach by Levin and Smith (1994) buyers are assumed to know only the distribution of their valuations at the time of entering the market. An alternative assumption would be that buyers know their valuations before entering the market (see Samuelson 1985). The appropriate assumption is an empirical matter, and depends on the analyzed market. Marmer, Shneyerov, and Xu (2007) have recently developed a nonparametric test for randomized entry. Their empirical application concerns bidding data for surface paving and grading contracts let by the Oklahoma Department of Transportation during the period Jan 02 - Dec 05. Their findings lend support to the Levin and Smith's (1994) hypothesis of randomized entry over that of selective entry according to Samuelson's (1985) model.

${ }^{4}$ In this framework the revelation principle applies. Therefore, without loss of generality, I will consider direct revelation mechanisms only.

${ }^{5}$ By ordered vector $x^{s}$ I refer to the vector of valuations of the bidders from a subset $s$, in which the components are ordered in an ascending order according to the bidder's number.

${ }^{6}$ Note that the valuation of each bidder $i, x_{i}$, might or might not appear in the vector $x$ depending on whether this bidder participates in the mechanism or not.
} 
and require the allocation and payment rules to satisfy conditions $(P),(F),(A N)$ and $(I C)$ given below. The mechanism of seller $j$ is denoted by $A_{j}$, and the vector of the mechanisms of all sellers by $A=\left(A_{1}, A_{2}, \ldots, A_{J}\right)$.

- (P) Participation. $p_{i}\left(x^{s}\right)=0, z_{i}\left(x^{s}\right)=0, \forall i \notin s$. Only buyers who participate in a certain mechanism can win the object and be required to pay.

- (F) Feasibility. $\sum_{i=1}^{I} p_{i}(x) \leq 1, \forall x \in X$. The allocation rule does not allow more units to be sold than physically available for any realization of buyer valuations. ${ }^{7}$

- (AN) Anonymity. Sellers do not discriminate among buyers on characteristics different than their bids. In other words, the chances of winning and the payment cannot depend on the buyers' identities but solely on their bids. Formally, the functions $p$ and $z$ are required to be permutation invariant. This means permuting the valuations of any ordered vector $x \in X$ permutes the vectors $p(x)$ and $z(x)$ in the same fashion. ${ }^{8}$

- (IC) Incentive compatibility:

Assume bidder $i$ participates in mechanism $A_{j}$. At the time of bidding, he knows his valuation $x_{i}$ and the set of bidders who participate in the same mechanism. Because I require mechanisms be anonymous, the probability of winning the item, and the payment of bidder $i$ depend only on the number of his rivals, and not on their identity. Let the number of all bidders visiting seller $j$ be $n$. If bidder $i$ reports the valuation $\tilde{x}_{i}$, and all other bidders report truthfully, the expected probability of winning the

\footnotetext{
${ }^{7}$ For some realizations of $x$ the inequality is allowed hold. The strict inequality would hold, for instance, when the mechanism is a second-price auction with a positive reservation price. In this case, if the valuation of the participating bidders are below the seller's reserve price, the seller will retain the item.

${ }^{8}$ Let $\left(p_{1}(\cdot), p_{2}(\cdot), \ldots, p_{I}(\cdot)\right)$ and $\left(z_{1}(\cdot), z_{2}(\cdot), \ldots, z_{I}(\cdot)\right)$ denote the vectors of probability and allocation functions, respectively. Let $\left(x_{k}, x_{l}, x^{s}\right)$ denote the ordered vector of valuations of the bidders from the group $s$ and the bidders $l, k \notin s$. Permutation invariance implies:

$$
\begin{aligned}
p_{k}\left(x_{k}, x_{l}, x^{s}\right) & =p_{l}\left(x_{l}, x_{k}, x^{s}\right) ; z_{k}\left(x_{k}, x_{l}, x^{s}\right)=z_{l}\left(x_{l}, x_{k}, x^{s}\right) ; \\
p_{i}\left(x_{k}, x_{l}, x^{s}\right) & =p_{i}\left(x_{l}, x_{k}, x^{s}\right) ; z_{i}\left(x_{k}, x_{l}, x^{s}\right)=z_{i}\left(x_{l}, x_{k}, x^{s}\right), \forall l, k, \forall i \in s, \forall s .
\end{aligned}
$$

The anonymity condition is sometimes called "equal treatment"; see McAfee (1993) and Peters (1994) for equivalent formulations.
} 
item and the expected payment will respectively be

$$
\begin{aligned}
P_{A_{j}}^{(n)}\left(\tilde{x}_{i}\right) & :=\int p_{i}\left(\tilde{x}_{i}, x^{(n-1)}\right) d F\left(x^{(n-1)}\right), \\
Z_{A_{j}}^{(n)}\left(\tilde{x}_{i}\right) & :=\int z_{i}\left(\tilde{x}_{i}, x^{(n-1)}\right) d F\left(x^{(n-1)}\right),
\end{aligned}
$$

where $x^{(n-1)}$ is the vector of other bidders' bids, $p_{i}\left(\tilde{x}_{i}, x^{(n-1)}\right)$ is the allocation rule, and $z_{i}\left(\tilde{x}_{i}, x^{(n-1)}\right)$ is the payment rule of mechanism $A_{j}$. The incentive compatibility condition states bidder $i$ finds it profitable to report truthfully if all other bidders do so, i.e. the following inequality ${ }^{9}$ holds $^{10}$ for every $n$ and every $\tilde{x}_{i} \in[0,1]$ :

$$
\begin{aligned}
E_{A_{j}}^{(n)}\left(\tilde{x_{i}} \mid x_{i}\right) & =: x_{i} \cdot P_{A_{j}}^{(n)}\left(\tilde{x}_{i}\right)-Z_{A_{j}}^{(n)}\left(\tilde{x}_{i}\right) \\
& \leq x_{i} \cdot P_{A_{j}}^{(n)}\left(x_{i}\right)-Z_{A_{j}}^{(n)}\left(x_{i}\right) \equiv E_{A_{j}}^{(n)}\left(x_{i} \mid x_{i}\right)=: E_{A_{j}}^{(n)}\left(x_{i}\right) .
\end{aligned}
$$

In this model, the individual rationality condition will be contained in the equilibrium concept where buyers' entry decision is modelled.

\subsection{Buyer strategy space}

Conditional on observing the mechanisms on offer, each buyer chooses which seller to go to. Buyers randomize across the set of sellers and the option to stay out of the market. A buyer's strategy, $m^{i}: \mathfrak{A}^{J} \rightarrow \Delta(J \cup o)$ is a mapping from the set of sellers' trade mechanisms into the set of probability distributions over the sellers, $J$, and the option to staying out of the market denoted by $0 .{ }^{11}$

\footnotetext{
${ }^{9} E_{A_{j}}^{(n)}\left(\tilde{x}_{i} \mid x_{i}\right)$ is the expected payment of a bidder, who has a valuation of $x_{i}$ and reports the valuation $\tilde{x}_{i}$ in the mechanism $A_{j}$.

${ }^{10}$ The requirement that sellers use incentive compatible mechanisms is without loss of generality. In the present setting buyers submit bids after they learn their valuations and the number of their fellow bidders (but not their valuations). Thus, sellers' mechanisms described here are standard Bayesian games for which the revelation principle applies (see Myerson 1997, p. 260).

${ }^{11}$ Buyers indeed play a behavior strategy because at every "node" (or subgame) defined by a profile of trade mechanisms, they choose the probability of visiting each seller (and the probability of staying out of the market).
} 


\subsection{Payoffs}

As I focus on anonymous mechanisms, only the number, but not identity, of the rivals of bidder $i$ matters for his payoff. When bidder $i$ visits seller $j$ with a probability of one, and all other bidders visit this seller with a probability of $m$, the expected payoff of bidder $i$ is given by

$$
R_{j}^{i}\left(A_{j} ; m\right)=\sum_{n=1}^{I} \operatorname{Pr}[n-1 ; m] \cdot \int_{0}^{1} E_{A_{j}}^{(n)}\left(x_{i}\right) d F\left(x_{i}\right)-c
$$

where

$$
\operatorname{Pr}[n-1 ; m]=\sum_{n=1}^{I}\left(\begin{array}{l}
I-1 \\
n-1
\end{array}\right) m^{n-1}(1-m)^{I-n}
$$

is the binomial probability with which bidder $i$ faces $(n-1)$ rivals. If all buyers go to seller $j$ with a probability of $m$, the expected payoff of seller $j$ is

$$
\Pi_{j}\left(A_{j} ; m\right)=\operatorname{Pr}[n ; m] \cdot \int_{0}^{1} Z_{j}^{(n)}\left(x_{i}\right) d F\left(x_{i}\right)
$$

where

$$
\operatorname{Pr}[n ; m]=\left(\begin{array}{l}
I \\
n
\end{array}\right) m^{n}(1-m)^{I-n}
$$

and the probability that exactly $n$ bidders go to seller $j$. The strategy of bidder $i$ is denoted by

$$
m^{i}(\cdot)=\left(m_{0}^{i}(\cdot), m_{1}^{i}(\cdot), m_{2}^{i}(\cdot) \ldots, m_{J}^{i}(\cdot)\right) .
$$

To economize on notation, let all other bidders play the (same) mixed strategy

$$
m^{-i}(\cdot)=\left(m_{0}^{-i}(\cdot), m_{1}^{-i}(\cdot), m_{2}^{-i}(\cdot) \ldots, m_{J}^{-i}(\cdot)\right) \cdot{ }^{12}
$$

Given sellers play the strategy profile $A$, the expected payoff of bidder $i$ is given by ${ }^{13}$

\footnotetext{
${ }^{12}$ Here $m_{0}^{i}(\cdot)$ is the probability with which bidder $i$ does not enter the market, and $m_{0}^{-i}(\cdot)$ is the probability with which another bidder does not enter the market. $m_{j}^{i}(\cdot)$ and $m_{j}^{-i}(\cdot)$ are the probabilities, with which bidder $i$ and another bidder, respectively, go to seller $j=1,2, \ldots, J$.

${ }^{13}$ The expected payoff of each bidder can be defined in a similar way when other bidders play asymmetric (mixed) strategies. In this case the expected payoff $R_{j}^{i}\left(A_{j} ; \cdot\right)$ will depend on the probabilities with which each rival of bidder $i$ visits seller $j$. To conserve space I do not introduce these payoffs here as they are not needed for the analysis of the symmetric equilibrium.
} 


$$
E R_{i}\left(A ; m^{i}(A), m^{-i}(A)\right)=\sum_{j=1}^{J} m_{j}^{i}(A) \cdot\left(R_{j}^{i}\left(A_{j} ; m_{j}^{-i}(A)\right)-c\right)
$$

\subsection{Equilibrium}

I now define the symmetric subgame perfect equilibrium for this decentralized trading game.

Definition 1 The sellers' strategy profile $A^{*}$ and the (behavioral) bidder strategy profile $m^{*}(\cdot)$ constitute a (symmetric subgame perfect) equilibrium, if they satisfy the following conditions:

Buyer behavior:

(B1) Buyers play a symmetric mixed strategy Nash equilibrium in every subgame $A$.

$$
\begin{gathered}
E R_{i}\left(A ; m^{* i}(A), m^{*-i}(A)\right) \geq E R_{i}\left(A ; m^{i}, m^{*-i}(A)\right), \forall A \in \mathfrak{A}^{J}, \forall i, \forall m^{i} \in[0,1], \\
m^{* i}(A)=m^{*-i}(A), \forall A \in \mathfrak{A}^{J}, \forall i
\end{gathered}
$$

(B2) Buyers do not change their distribution across sellers unless a seller deviates to a mechanisms that offers them a higher or a lower payoff. That is, for all $\tilde{A}_{j}$ such that $R_{j}^{i}\left(\tilde{A}_{j} ; m_{j}^{*}\left(A_{j}, A_{-j}\right)\right)=R_{j}^{i}\left(A_{j} ; m_{j}^{*}\left(A_{j}, A_{-j}\right)\right)$, the equality $m_{j}^{*}\left(\tilde{A}_{j}, A_{-j}\right)=$ $m_{j}^{*}\left(A_{j}, A_{-j}\right)$ holds.

Seller behavior:

(S1) Sellers play a symmetric Nash equilibrium in the first stage of the game.

$$
\begin{gathered}
\Pi_{j}\left(A_{j}^{*}, A_{-j}^{*} ; m^{*}(\cdot)\right) \geq \Pi_{j}\left(A_{j}, A_{-j}^{*} ; m^{*}(\cdot)\right), \forall A_{j} \in \mathfrak{A} . \\
A_{1}^{*}=A_{2}^{*}=\cdots=A_{J}^{*} .
\end{gathered}
$$

Equilibrium behavior prescribes that sellers choose the same trade mechanism, and buyers distribute across sellers in the same fashion for each $A$. This continuation equilibrium is appealing at least for three reasons. First, it guarantees a symmetric treatment of buyers. Requiring symmetry appears quite intuitive because buyers are assumed to be 
ex ante identical and anonymous, and there is no device in this game allowing them to coordinate. Second, the number of bidders in each auction will be stochastic, and this is a phenomenon frequently observed in practice. Finally, the probability of entry changes continuously with the mechanisms' variables, e.g. entry fees or reserve prices. This will be helpful for the characterization of equilibrium.

\section{Organization of the analysis and results}

The first step of the analysis is to explore what kind of trade mechanisms could possibly be equilibria. Theorem 1 provides an answer to this question by asserting that in equilibrium (if one exists) sellers hold auctions. Holding an auction in this type of model actually means using a trade mechanism which assigns the good to the participant with the highest valuation (for a similar interpretation see McAfee 1993, p. 1292). Note the Revenue Equivalence Theorem applies here because by assumption buyer valuations are private, symmetric and independent. Hence, the equilibrium auctions will not be uniquely defined (e.g. sellers can use first price, second price, an all pay auction, etc.). Table 1 provides a useful illustration of the remaining results and arguments. The rows in this table present conditions on trade mechanisms which define the sets $\mathfrak{A}, \Omega$ and $\Omega_{e}$. The first three conditions were already defined in the previous section. "Efficiency" requires the object be always granted to the participant with the highest valuation; "Constant entrance fee" requires the use of an auction with an entrance fee that does not depend on the number of actual bidders.

\begin{tabular}{c|c|c|c|} 
& $\mathfrak{A}$ & $\Omega$ & $\Omega_{e}$ \\
\hline Feasibility & + & + & + \\
\hline Anonymity & + & + & + \\
\hline Incentive Compatibility & + & + & + \\
\hline Efficiency & & + & + \\
\hline Constant entry fee & & & + \\
\hline
\end{tabular}

Table 1: Mechanism sets and conditions. The + sign denotes the conditions satisfied by the mechanisms from the sets $\mathfrak{A}$ (strategy set of the original game), $\Omega$ (auctions with zero reserve price and entry fees that might depend on the number of bidders) and $\Omega_{e}$ (auctions with zero reserve price and entry fee independent of the number of actual bidders).

Let us consider mechanisms giving every buyer a constant expected payment when all buyers visit this mechanism with a certain constant probability (say $m$ ). Lemma 1 states 
that among all these mechanisms, the ones maximizing seller's expected payoff must satisfy the "Efficiency" condition. This argument narrows down the set of mechanism that can constitute an equilibrium, and upon considering the incentive compatibility constraint, only the mechanisms from the set $\Omega$ remain possible equilibrium candidates. That is, the equilibrium trade mechanisms can only be auctions with a zero reserve price, and an entrance fee that might depend on the number of the participating bidders.

Let us focus now on the subset $\Omega_{e}$ consisting only of auctions with a uniform entrance fee (i.e. entrance fee independent of the number actual bidders). Theorem 2 claims every equilibrium of the game with strategy $\Omega_{e}$ is also an equilibrium of the game with the larger strategy set $\Omega$. This result allows me to focus on the game with strategy set $\Omega_{e}$, in which sellers choose a single variable-the entry fee in their auction. Theorem 3 then provides an explicit solution for the entry fee of the unique equilibrium of the game with strategy set $\Omega_{e}$. This is also an equilibrium of the original game formulated in the model.

The essence of the analysis presented in Theorem 3 is best conveyed by considering the case of two sellers and two or more buyers. Assume the cost of entering the market is zero and two sellers hold auctions with an entry fee of $C$. In the continuation equilibrium, buyers randomize equally between visiting the two sellers. When one of the sellers, say seller $j$, raises his fee to $C_{j}$, this seller will see an outflow of buyers because customers will redistribute across the two sellers in a way that their expected gain is equal at each seller. Note that the reduction in the probability with which buyers visit seller $j$ depends only on the difference between $C_{j}$ and $C$ rather than on their actual values. By raising his entry fee beyond $C$, seller $j$ faces a tradeoff. On the one hand, he is able to extract an additional profit from bidders who stay in the auction in the form of increased entry fees. ${ }^{14}$ On the other hand, he loses profit from bidders who exit the auction. For a fixed difference $C_{j}-C$, and thus fixed outflow of buyers, the loss of profit increases in $C$ because the buyers who leave the auction are not paying this entry fee anymore. The equilibrium level of the entry fee is such that, for entry fees $C_{j}$ higher than this level, the loss incurred by the outflow of buyers exceed the benefit; and for entry fees $C_{j}$ lower than this level the benefit from the influx of additional buyers is not sufficient to compensate for the loss associated with the reduction of the entry fee for all participants. The proof of Theorem 3 makes this argument

\footnotetext{
${ }^{14}$ There is a secondary effect that goes in the opposite direction. It is caused by the reduced buyer competition in the auction due to the outflow of buyers. This effect is smaller than the one incurred by the increased entry fee, and generally the consumer surplus decreases if one of the seller raises his fee. This effect does not play a role for the arguments that follow.
} 
precise and obtains an explicit solution. This proof relies on an innovation that might be applicable in related auction games with random entry. Rather than formulating the payoff of seller $j$ as a function of the entry fees $C$ and $C_{j}$, this payoff is expressed in terms of $C$ and the probability $m$ with which seller $j$ is visited. This formulation allows me to link the total surplus of all buyers and seller $j$ to the expected payoff of an individual buyer (Lemmas 3 and 4$)$. This yields a convenient representation of seller $j$ 's payoff, $\Pi_{j}(m, C)$. Then, the equilibrium entry fee $C$ is determined by the condition that $\Pi_{j}(m, C)$ reaches a maximum when buyers visit each seller with the same probability (with two sellers $m=1 / 2$, which implies $C_{j}=C$ ).

The rest of the analysis provides some comparative static results. Theorem 4 states that the equilibrium entry fee converges to zero as the size of the market increases. Hereby I let the number of agents go to infinity, but keep the ratio of buyers to sellers constant. A similar approach is taken in Theorem 5, which shows that buyers obtain a higher surplus than sellers in equilibrium if the market is large. In particular, I obtain a closed form solution for the buyer and seller surplus when the buyer valuations are uniformly distributed, and the number of agents converges to infinity.

\section{Theorems and proofs}

The first theorem narrows down the set of mechanisms that can potentially be equilibria.

Theorem 1 (i) In equilibrium, sellers use mechanisms that assign the item to the highestvaluation bidder, if this valuation is higher than seller's use value of the good.

(ii) The equilibrium mechanisms are (expected) payoff equivalent to a second-price auction with a reserve price equal to seller's use value, and an entrance fee, which might depend on the number of participating bidders.

Proof. The proof of part (i) of the theorem begins with the following lemma.

Lemma 1 Consider the mechanisms, which give every bidder an expected payoff of $R$, provided all bidders visit this mechanism with a probability of $m$. Among these mechanisms, the ones that maximize the expected payoff of the seller, assign the object with a probability of one to the participant with the highest valuation, provided that this valuation exceeds the use value of the seller. 
The sketch of the proof will be briefly illustrated here (see the Appendix for details). The expected payoff of the seller is the joint surplus of all agents (the sum of the payoffs of all bidders and the seller) minus the expected payoff of the bidders, which is assumed constant, and equals $I \cdot m \cdot R$. Therefore, to maximize his expected payoff, the seller should maximize the expected joint surplus resulting from trade (the surplus of all bidders and the seller). For any number of buyers, the expected joint surplus is the sum of the bidder valuations multiplied by their probabilities of winning the item. Obviously, this surplus is maximized when the good is always assigned to the highest valuation bidder with a probability of one. ${ }^{15}$

Now let $n \in\{1,2, \ldots, I\}$ be the total number of bidders visiting a certain seller $j$. Let mechanism $A_{j}$ assign the good to the highest valuation bidder, and let this mechanism be incentive compatible. The winning probability of a bidder with a valuation $x_{i}$ who reports a valuation of $\tilde{x}_{i}$ is $P_{i}^{(n)}\left(\tilde{x}_{i}\right) \equiv\left[F\left(\tilde{x}_{i}\right)\right]^{n-1}$. The incentive compatibility condition (IC) requires $\left.\frac{d}{d x_{i}}\left(x_{i} \cdot P_{A_{j}}^{(n)}\left(\tilde{x}_{i}\right)-Z_{A_{j}}^{n}\left(\tilde{x_{i}}\right)\right)\right|_{\tilde{x}_{i}=x_{i}}=0$, and applying the Envelope Theorem, I obtain $\frac{d}{d x_{i}}\left(E_{A_{j}}^{(n)}\left(\tilde{x}_{i} \mid x_{i}\right)=\left.\frac{\partial}{\partial x_{i}}\left(E_{A_{j}}^{(n)}\left(\tilde{x}_{i} \mid x_{i}\right)\right)\right|_{\tilde{x}_{i}=x_{i}}=\left[F\left(x_{i}\right)\right]^{n-1}\right.$. The expected profit of a bidder with a valuation of $x_{i}$ is thus

$$
E_{A_{j}}^{(n)}\left(x_{i} \mid x_{i}\right)=\left(-C_{n}\right)+\int_{0}^{x_{i}}[F(z)]^{n-1} d z
$$

where $\left(-C_{n}\right)$ is the expected profit of a bidder with the lowest valuation $\left(x_{i}=0\right)$. The amount $C_{n}$ is the entrance fee that each bidder has to pay when participating with $(n-$ 1) other bidders (if $C_{n}$ is negative, the seller is actually giving a participation bonus to bidders). If there is no entry fee or bonus, the ex-ante payoff of a bidder is

$$
B_{n}=\int_{0}^{1} \int_{0}^{x}[F(z)]^{n-1} d z d F(x) .
$$

A standard result in auction theory (see e.g. Riley and Samuelson 1981, McAfee and McMillan 1987a) establishes that a seller, who auctions off an item to $n$ bidders, receives an expected payoff equal to the expected value of the second order statistic given by

\footnotetext{
${ }^{15}$ McAfee and McMillan (1987b) provide a similar argument in a setting with one seller and an outside option. For any number of participating bidders, seller's expected revenue is the winning bidder's expected valuation minus the expected profit of the participating bidders. Thus, for any number of bidders, the seller should award the good so as to maximize the expected valuation of the winner. This can only be done by always assigning the good to the highest valuation bidder.
} 


$$
S_{n}=n \cdot \int_{0}^{1}[x \cdot f(x)+F(x)-1] \cdot[F(x)]^{n-1} d x .
$$

A mechanism from the set $\Omega$ can now be identified by the participation fees $C_{1}, C_{2}, \ldots, C_{I}$ in the cases of $1,2, \ldots$, and $I$ participating bidders, respectively.

Let $A_{j}^{C} \in \Omega_{e}$ be a revenue equivalent mechanism to a second price auction with an entry fee of $C$ (independent of the number of participants).

Theorem 2 If the seller strategy profile $A^{C}=\left(A_{1}^{C}, A_{2}^{C}, \ldots, A_{I}^{C}\right)$ is an equilibrium of the game with a strategy space $\Omega_{e}$, (i.e. the game in which sellers compete by setting a constant entry fee in their auctions), then it is an equilibrium of the game with a strategy set $\Omega$ (the game in which sellers can condition their entry fee on the number of participants).

The idea of the proof is simple, and it will shortly be presented here. A detailed proof can be found in the Appendix. Assume by contradiction that $A^{C}$ is not an equilibrium of the game in which sellers can condition their entry fee on the number of participants (the game with strategy set $\Omega$ ). Then, a deviation mechanism $\tilde{A}_{j}$ for a certain seller $j$ exists, which gives this seller a higher expected payoff. Let buyers visit this seller with a probability of $\tilde{m}$. Given this probability, the expected payment of a bidder can be divided into an expected payment arising from his participation in the auction and an expected entry fee payment. Construct now a new mechanism, $A_{j}^{\tilde{C}}$, which charges a constant entry fee equal to the expected entry fee payment of $\tilde{A}_{j}$. The mechanisms $A_{j}^{\tilde{C}}$ and $\tilde{A}_{j}$ are auctions with entry fees such that buyers are paying on average the same entry fee (if they visit seller $j$ with a probability of $\tilde{m}$ ). Applying condition (B2) stated in the equilibrium definition, I conclude that bidders visit seller $j$ with a probability of $\tilde{m}$ when seller $j$ uses either $\tilde{A}_{j}$ or $A_{j}^{\tilde{C}}$. That means $A_{j}^{\tilde{C}}$ and $\tilde{A}_{j}$ generate the same expected payoff to the seller. Because $\tilde{A}_{j}$ was a profitable deviation for seller $j$, playing $A_{j}^{\tilde{C}}$ must be more profitable for seller $j$ compared to playing $A_{j}^{C}$. This argument delivers the desired contradiction to the assumption that $A_{j}^{C}$ is an equilibrium mechanism and completes the proof.

This theorem has a merit on its own as its validity goes beyond the scope of the present setting. The only assumption needed for the proof is risk neutrality, and the theorem holds in private and common value environments in which bidder valuations might possibly be interdependent. In this model the theorem allows me to focus on the game in which sellers 
compete by setting entry fees independent of the number of bidders. The next theorem characterizes the equilibrium entry fee.

Theorem 3 In equilibrium, sellers hold auctions with an entry fee. The entry fee is uniquely determined by the number of buyers and sellers, the distribution of buyer valuations, and the cost of entering the market. Let

$$
R[m]:=\sum_{n=1}^{I} \operatorname{Pr}[n-1 ; m] \cdot B_{n}
$$

be the payoff of a bidder who participates in an auction with no entry fee or reserve price, provided all other bidders participate in the same auction with a probability of $m$; and let

$$
E:=\left.\frac{1}{J} \cdot \frac{d}{d m}\left(R\left[\frac{1-m}{J-1}\right]\right)\right|_{m=1 / J} .
$$

The equilibrium entry fee is given as follows:

(1) $c<R[1 / J]-E$. Sellers use an entry fee equal to E. All buyers enter the market with a probability of one and receive an expected payoff in excess of their cost.

(2) $R[1 / J]-E \leq c<R[1 / J]$. All buyers enter the market with a probability of one and just cover their cost of entry. All sellers use an entry fee of $R[1 / J]-c>0$. As the cost of entering the market (c) increases, all sellers lower their entry fees so as to allow bidders to enter the market with a probability of one.

(3) $c \geq R[1 / J]$. Buyers enter the market with a probability smaller than one. The entry fee in the equilibrium auctions is zero. In particular, if $c \geq R[0]$, buyers stay out of the market with a probability of one.

The graph below illustrates how the entry fee depends on the buyer cost of entering the market for a given distribution of buyer valuations and a given number of buyers and sellers.

For small values of the entry cost $c$, sellers charge an entry fee of $E$ and buyers enter with a probability of one. As the cost of entry increases, sellers collectively lower the entry fee so as to allow buyers to enter with a probability of one. This pattern continues until the cost of entering the market becomes so large that sellers reduce their entry fees to zero. When $c$ rises further, sellers keep their entry fees at zero, but buyers start leaving 


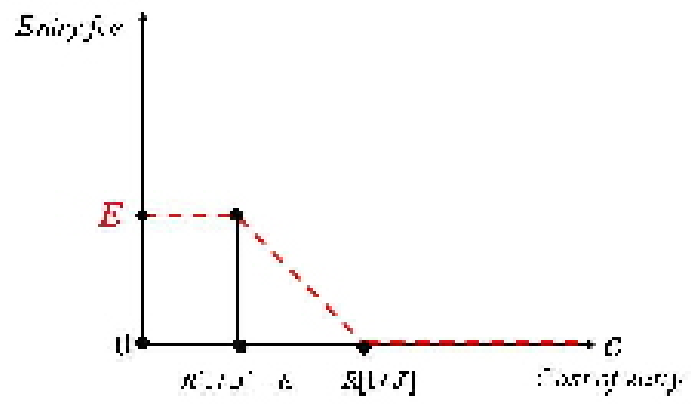

Figure 1: Equilibrium entry fee as a function of the buyer cost of entering the market.

the market with a positive probability. When the entry cost reaches the critical level $R[0]$, buyers stay out of the market completely.

Proof of Theorem 3.

Let seller $j$ charge an entry fee of $C_{j}$ and let all other sellers charge an entry fee of $C$. The expected profit of seller $j$ is given by

$$
\Pi_{j}\left(C_{j}, C\right)=\sum_{n=1}^{I} \operatorname{Pr}[n ; m] \cdot S_{n}+I \cdot m \cdot C_{j},
$$

where $m$ is the probability that buyers go to seller $j$ given the entry fees $C_{j}$ and $C$. How does the probability $m$ depend on the entry fees $C_{j}$ and $C$ ? Recall that $R[m]$ is the expected payoff of a bidder who participates in an auction without an entry fee or a reserve price, provided the other bidders enter the same auction with a probability of $m$. In the symmetric independent private value model, the function $R[m]$ is decreasing in $m$. That is, the higher the probability that other bidders attend the same auction, the lower the expected surplus of each individual bidder. ${ }^{16}$ This property of the bidder payoff ensures

\footnotetext{
${ }^{16}$ To prove this consider an auction with no entry fee or a reserve price in which bidder $i$ participates with a probability of one. Let all other bidders participate with a probability of $m$, except for one of them,
} 
that for each combination of entry fees charged by sellers, buyers distribute across sellers in a unique way. Let me break down all possible scenarios for the distribution of buyers across sellers depending on the entry fees in three categories.

Case A. All buyers enter the market with a probability of one and distribute across all sellers. In this case the entry probability $m$ is determined by the equilibrium condition

$$
R[m]-C_{j}=R\left[\frac{1-m}{J-1}\right]-C,
$$

if this equation has a solution $m \in[0,1]$, and for this solution the inequality $R[m]-C \geq$ $c$ holds. That is, buyers find it profitable to enter the market as they earn a surplus not lower than their cost of entry. Buyers visit seller $j$ with a probability of $m$ and each of the other sellers with a probability of $\frac{1-m}{J-1}$. Equation (A) ensures that each buyer earns the same surplus by going to any of the sellers given the randomization strategy of the other buyers.

Case B. Buyers enter the market with a probability smaller than one. This is the case when equation $(A)$ has a solution $m \in[0,1]$, but for this solution $R[m]-C<c$. In this case buyers visit seller $j$ with a probability determined by the equation

$$
R[m]-C_{j}=c
$$

if this equation has a solution in the interval $[0,1]$.

Case C. Consider the constellations of $C_{j}$ and $C$ for which equation (A) is not satisfied for $m \in[0,1]$. I will discuss in turn the two possible cases in which the solution of equation $(\mathrm{A})$ is either greater than one or below zero.

(C1) Equation (A) has a solution $m>1$. Seller $j$ charges such a low entry fee that buyers prefer to visit this seller with a probability of one rather than to visit any of the other sellers (i.e. the inequality $R[1]-C_{j}>R[0]-C$ holds). Three cases now are possible depending on the solution of equation (B).

named $k$, who participates with a probability of $m_{k}$. The payoff of bidder $i$ is $R\left[m_{k}, m\right]=\sum_{n=0}^{I-2} \operatorname{Pr}[n ; m]$. $\left(m_{k} \cdot B_{n+1}+\left(1-m_{k}\right) B_{n}\right)$. The derivative with respect to the entry probability of the other bidders is $\frac{d R[m]}{d m}=\left.(I-1) \cdot \frac{\partial R\left[m_{k}, m\right]}{\partial m_{k}}\right|_{m_{k}=m}=(I-1) \cdot \sum_{n=0}^{I-2} \operatorname{Pr}[n ; m]\left(B_{n+1}-B_{n}\right)<0$, where $B_{n}$ is the expected surplus of a bidder who participates in an auction with $(n-1)$ other bidders, and this expected surplus decreases in the the number of rivals $(n-1)$. The derivative is negative because $B_{n+1}<B_{n}$ for all $n$. 
C1.1. Equation (B) has a solution $m \in[0,1]$. Buyers randomize between not entering the market and going to seller $j$.

C1.2. Equation (B) has a solution $m>1$. Buyers go to seller $j$ with a probability of one and earn a higher surplus than their cost of entry (i.e. the inequality $R[1]-C_{j}>c$ holds).

C1.3. Equation (B) has a solution $m<0$. Seller $j$ receives no customers (i.e. the inequality $R[0]-C_{j}<c$ holds).

(C2) Equation (A) has a solution $m<0$. Seller $j$ charges a very high entry fee and receives no buyers (the inequality $R[0]-C_{j}<R[0]-C$ holds). Customers do not enter the market.

The analysis focuses on the symmetric equilibrium - the equilibrium in which sellers charge the same entry fee, and buyers randomize symmetrically across sellers. To derive this equilibrium, I need to determine an entry fee $C$ such that, if all sellers charge this entry fee, no deviation of a single seller $C_{j}$ exists, which increases the expected payoff of this seller. The next lemma demonstrates that, for the purpose of the equilibrium analysis, it is sufficient to analyze only the deviations $C_{j}$ for which either equation (A) or equation (B) has a solution $m \in[0,1]$.

Lemma 2 Let all sellers charge an entry fee $C$. For any deviation $C_{j}$ of a single seller, for which the equations $(A)$ and $(B)$ do not have a solution $m \in[0,1]$, there exists a weakly more profitable deviation $\tilde{C}_{j}$ of this seller $\left(\right.$ i.e. $\left.\Pi_{j}\left(\tilde{C}_{j}, C\right) \geq \Pi_{j}\left(C_{j}, C\right)\right)$ for which at least one of the equations $(A)$ or $(B)$ has a solution $m \in[0,1]$.

The proof of the lemma is rather straightforward. The only cases for which neither (A) nor (B) holds are the cases C1.2., C1.3. and (C2). In case C1.2. seller $j$ leaves too much surplus to buyers. Buyers go to this seller with a probability of one and earn a surplus in excess of their cost. In this case seller $j$ can raise his entry fee to the point until one of the equations (A) or (B) holds for $m=1$ without losing buyers and increase his expected profit. In case C1.3. seller $j$ receives no customers and earns a zero profit. In this case, lowering $C_{j}$ in a way that the equality $R[0]-C_{j}=c$ holds will lead to the same expected profit of zero for this seller. In case (C2), seller $j$ also receives no customers. In this case, lowering $C_{j}$ so that either equation (A) or equation (B) is satisfied for $m=0$ will not reduce the 
payoff of seller $j$ as this seller will still not be visited by buyers. These arguments establish the stated result.

Because of this statement, it will be sufficient to describe the sellers' payoffs only for cases in which $m$ behaves according to equation (A) or equation (B). When seller $j$ chooses an entry fee $C_{j}$, seller $j$ indirectly determines the entry probability $m$. Therefore, the decision of seller $j$ can alternatively be viewed as a choice of the entry probability $m$, whereby the entry fee $C_{j}$ is indirectly determined from equation (A) or equation (B). It will be convenient to express the payoff of seller $j$ as a function of $m$ and $C$.

$$
\Pi_{j}(m, C)=\left\{\begin{array}{ll}
\sum_{n=1}^{I} \operatorname{Pr}[n ; m] \cdot S_{n}+I \cdot m \cdot\left(R[m]-R\left[\frac{1-m}{J-1}\right]+C\right) & \text { if eq. (A) holds, } \\
\sum_{n=1}^{I} \operatorname{Pr}[n ; m] \cdot S_{n}+I \cdot m \cdot(R[m]-c) & \text { if eq. (B) holds. }
\end{array} \Leftrightarrow\right.
$$

$\Pi_{j}(m, C)= \begin{cases}\sum_{n=1}^{I} \operatorname{Pr}[n ; m] \cdot S_{n}+I \cdot m \cdot R[m]-I \cdot m \cdot\left(R\left[\frac{1-m}{J-1}\right]-C\right) & \text { if eq. (A) holds } \\ \sum_{n=1}^{I} \operatorname{Pr}[n ; m] \cdot S_{n}+I \cdot m \cdot R[m]-I \cdot m \cdot c & \text { if eq. (B) holds. }\end{cases}$

Let me define now the expected Joint Surplus of seller $j$ and all buyers who visit him with a probability of $m$ by

$$
J S(m):=\sum_{n=1}^{I} \operatorname{Pr}[n ; m] \cdot S_{n}+I \cdot m \cdot R[m]=\sum_{n=1}^{I} \operatorname{Pr}[n ; m] \cdot\left(S_{n}+n \cdot B_{n}\right),
$$

where the last expression is the weighted sum of the buyer and seller surplus for all possible numbers of participating buyers. The payoff of seller $j$ can be expressed as

$$
\Pi_{j}(m, C)= \begin{cases}J S(m)-I \cdot m \cdot\left(R\left[\frac{1-m}{J-1}\right]-C\right) & \text { if eq. (A) holds } \\ J S(m)-I \cdot m \cdot R[m]-I \cdot m \cdot c & \text { if eq. (B) holds. }\end{cases}
$$

Next, I make use of a well-known result in auction theory presented below.

Lemma 3 (Levin and Smith, 1994) In the symmetric independent private value model, when one bidder joins an auction in which $(n-1)$ bidders are participating (with a probability of one), the change in the Joint Surplus is equal to the individual bidder's gain:

$$
\left(S_{n}+n \cdot B_{n}\right)-\left(S_{n-1}+(n-1) \cdot B_{n-1}\right)=B_{n}
$$


A concise proof of this lemma can be found in Levin and Smith (1994, p. 592). This lemma is useful for the proof of the following statement.

Lemma 4 Assume all bidders visit a seller with a probability of $m$. The marginal change of the Joint Surplus equals the sum of all bidders' gains:

$$
\frac{d}{d m}(J S(m))=I \cdot R[m]
$$

The proof of the lemma is provided in the Appendix. In a symmetric equilibrium sellers use the same entry fee (say $C$ ) and this entry fee is such that if all other sellers charge $C$, it is optimal for seller $j$ to also charge the same fee. In other words, the equilibrium fee $C$ is such that $\Pi_{j}\left(C_{j}, C\right)$ is maximized for $C_{j}=C$. Here, the expected payoff of seller $j$ is expressed in terms of $C$ and $m$ (instead of $C_{j}$ ). Hence, in the case that equation (A) holds, the equilibrium condition can be reformulated to require that $C$ is such that $\Pi_{j}(m, C)$ is maximized for $m=1 / J$ (which implies $C_{j}=C$ ). ${ }^{17}$ Using the relationship presented in the previous lemma yields the following first order condition:

$$
\frac{d}{d m} \Pi_{j}(m, C)= \begin{cases}I \cdot R[m]-I \cdot \frac{d}{d m}\left(m \cdot\left(R\left[\frac{1-m}{J-1}\right]-C\right)\right)=0 & \text { if eq. (A) holds } \\ I \cdot R[m]-I \cdot c=0 & \text { if eq. (B) holds }\end{cases}
$$

Observe that for $m=1 / J$ the equality $R[m]=R\left[\frac{1-m}{J-1}\right]$ holds. If the entry probability is determined by equation (A), the solution of the first equation with respect to $C$ yields the equilibrium entry fee

$$
E=\left.\frac{1}{J} \cdot \frac{d}{d m}\left(R\left(\frac{1-m}{J-1}\right)\right)\right|_{m=1 / J}
$$

The first order condition in the case of equation (B) requires $R[m]-c=0$. Equation (B) itself requires $R[m]-C_{j}=c$, which combined with the previous equation yields $C_{j}=0 .{ }^{18}$ It remains to clarify now for which values of the entry cost $c$ the entry probability is determined by equation (A) and by equation (B).

\footnotetext{
${ }^{17}$ Analogously, in case B the equilibrium condition can be restated to require that $C$ be such that for the value of $m$ which maximizes $\Pi_{j}(m, C)$ equation $\mathrm{B}$ is satisfied.

${ }^{18}$ The convexity of $\Pi_{j}(m, C)$ in $m$ guarantees that the first order condition yields a unique maximum (recall that $R[m]$ is decreasing in $m$ ).
} 
Case 1. When all sellers charge $E$ and $c<R[1 / J]-E$, buyers indeed are willing to enter the market with a probability of one as they earn a higher surplus than their cost of entry. The entry probability is determined by equation (A). In this case, as has been shown, if all sellers charge $E$ it is optimal for seller $j$ to also use the same entry fee.

Case 2. When all sellers charge an entry fee of $R[1 / J]-c$ and $R[1 / J]-E \leq c<R[1 / J]$, buyers enter the market with a probability of one and are just able to cover their cost of entry. It will be shown that no deviation to a higher or a lower entry fee is profitable for seller $j$.

If seller $j$ deviates to an entry fee higher than $R[1 / J]-c$, buyer surplus from entering the market will decrease, and buyers will stay out of the market with positive probability. That means the entry probability $m$ and the entry fee $C_{j}$ will behave according to equation (B). In this case the payoff of seller $j$ decreases in $C_{j}$ (the maximum is attained when $C_{j}=0$ ). Raising $C_{j}$ beyond $R[1 / J]-c$ is thus not profitable for seller $j$.

If seller $j$ lowers $C_{j}$ below $R[1 / J]-c$ buyer surplus from entering the market will increase, and buyers will enter with a probability of one and realize a higher surplus than the entry cost. The entry probability $m$ and $C_{j}$ will behave according to equation (A). When $C_{j}=C=R[1 / J]-c<E$ buyers visit seller $j$ with a probability of $m=1 / J$, and the derivative of the expected payoff of seller $j$ in case $\mathrm{A}$ is

$$
\begin{aligned}
\frac{d}{d m} \Pi_{j}(m, C) \mid & \quad m=1 / J=I \cdot R[m]-\left.I \cdot \frac{d}{d m}\left(m \cdot\left(R\left[\frac{1-m}{J-1}\right]-C\right)\right)\right|_{m=1 / J} \\
= & I \cdot\left[R[m]-R\left[\frac{1-m}{J-1}\right]+C-\left.\frac{1}{J} \cdot \frac{d}{d m}\left(R\left(\frac{1-m}{J-1}\right)\right]\right|_{m=1 / J}\right. \\
= & I \cdot(C-E)<0 .
\end{aligned}
$$

That means raising $m$ above $1 / J$, or equivalently lowering $C_{j}$ below $R[1 / J]-c$, is also unprofitable for seller $j$.

Case 3. When $c \geq R[1 / J]$ and sellers charge an entry fee of zero, bidders enter the market with a probability smaller than one. The entry probability is determined by equation (B), and, as has been shown, the payoff of each seller $j$ is maximized for $C_{j}=$ 0 . 


\section{Comparative Statics}

In this section I will characterize the equilibrium entry fee in large markets, and will discuss this model relative to the literature on decentralized markets with an infinite number of buyers and sellers. I will also explore how the presence of more sellers impacts the entry fees used in equilibrium, and in particular how the monopoly case compares to the case of two sellers.

Following the approach by McAfee (1993) and Peters and Severinov (1997), I hold the ratio of buyers to sellers constant, $k=I / J$, and consider a sequence of markets for which $J=2,3, \ldots$ I focus on the interesting case in which buyer cost of entry is so low that in equilibrium no buyers stay out of the market.

Theorem 4 As the number of buyers and sellers converges to infinity, but their ratio remains constant, i.e. $I=k \cdot J$, the entry fee converges to zero:

$$
\lim _{J \rightarrow \infty} E_{J, k \cdot J}=0
$$

See the Appendix for a proof. This theorem demonstrates that a higher number of sellers leads to a more intense competition among sellers despite the proportionally increasing number of buyers. The intuition for this regularity is best conveyed by the following argument. When the number of sellers increases, the impact of each individual seller on the expected gain that buyers obtain at other sellers decreases. When the number of sellers is very large, this impact is very small, and if this effect is neglected entirely, the expected payoff of bidders who visit other sellers will be constant. A seller confronted with buyers who have a constant outside option optimally sets a zero entry fee (see e.g. Levin and Smith 1994). Ignoring this effect entirely is indeed at the core of the limiting equilibrium concepts proposed in McAfee (1993) and Peters and Severinov (1997). My analysis illustrates how the equilibrium entry fee diminishes when this effect decreases with the size of the market. The next table provides the entry fee $E_{J, k \cdot J}$ depending on the number of sellers for the case of uniformly distributed buyer valuations ${ }^{19}$ and markets with ratios $k=1,2$ and 3 .

\footnotetext{
${ }^{19}$ For a uniform distribution of buyer valuations it can easily be shown that $S_{n}=\frac{n-1}{n+1}, B_{n}=\frac{1}{n(n+1)}$ and $R[m]:=\sum_{n=1}^{I} \operatorname{Pr}[n-1 ; m] \cdot B_{n}$.
} 


\begin{tabular}{lccc}
\hline \# sellers & \multicolumn{3}{c}{ Ratio of buyers to sellers $\mathrm{k}=\mathrm{I} / \mathrm{J}$} \\
\hline & $\mathrm{k}=1$ & $\mathrm{k}=2$ & $\mathrm{k}=3$ \\
2 & 0.1667 & 0.2000 & 0.1473 \\
3 & 0.0830 & 0.0920 & 0.0701 \\
4 & 0.0552 & 0.0592 & 0.0456 \\
5 & 0.0412 & 0.0435 & 0.0338 \\
6 & 0.0328 & 0.0343 & 0.0268 \\
7 & 0.0273 & 0.0284 & 0.0222 \\
8 & 0.0233 & 0.0241 & 0.0189 \\
9 & 0.0203 & 0.0210 & 0.0164 \\
10 & 0.0181 & 0.0186 & 0.0146 \\
100 & 0.0016 & 0.0016 & 0.0013 \\
1000 & 0.0002 & 0.0002 & 0.0001 \\
$J \rightarrow \infty$ & 0 & 0 & 0 \\
\hline
\end{tabular}

Table 2: Equilibrium entry fee for uniformly distributed buyer values, different number of sellers, and different buyer/seller ratios.

The calculations are performed with Mathematica and the numbers are rounded to four decimal places. Let us turn now to the monopoly case. When $c>R[1]$ the monopolist's expected payoff is given by the function $\tilde{\Pi}_{j}\left(m, C_{j}\right)$, and, as already discussed (see Case 3 of Theorem 3), this function reaches its maximum for $C_{j}=0$ (for an analysis along similar lines see Levin and Smith 1994). When $c<R[1]$ buyers enter the market with a probability of one and earn a surplus of $R[1]-c$. In this case the seller can expropriate the entire surplus from buyers by charging an entry fee of $R[1]-c$. The graph below provides the entry fee in markets with 3 bidders, and a monopoly and duopoly seller market.

Interestingly, the availability of a second seller leads to higher entry fees. To gain intuition into this phenomenon it is useful to compare how raising the entry fee impacts the entry into a seller's mechanism. In the case of monopoly, the seller optimally charges an entry fee that expropriates the entire surplus from buyers. If the seller raises further his entry fee, buyers will start exiting the market. How is the duopoly situation different? If sellers use the same entry fee as in the monopoly case, buyers will distribute across the two sellers with a probability of $1 / 2$, and will face lower competition from other buyers. Their expected payoff will be higher. Thus, if one of the sellers slightly raises his entry fee, buyers will not exit the market, but will shift probability towards visiting the other seller. As a 


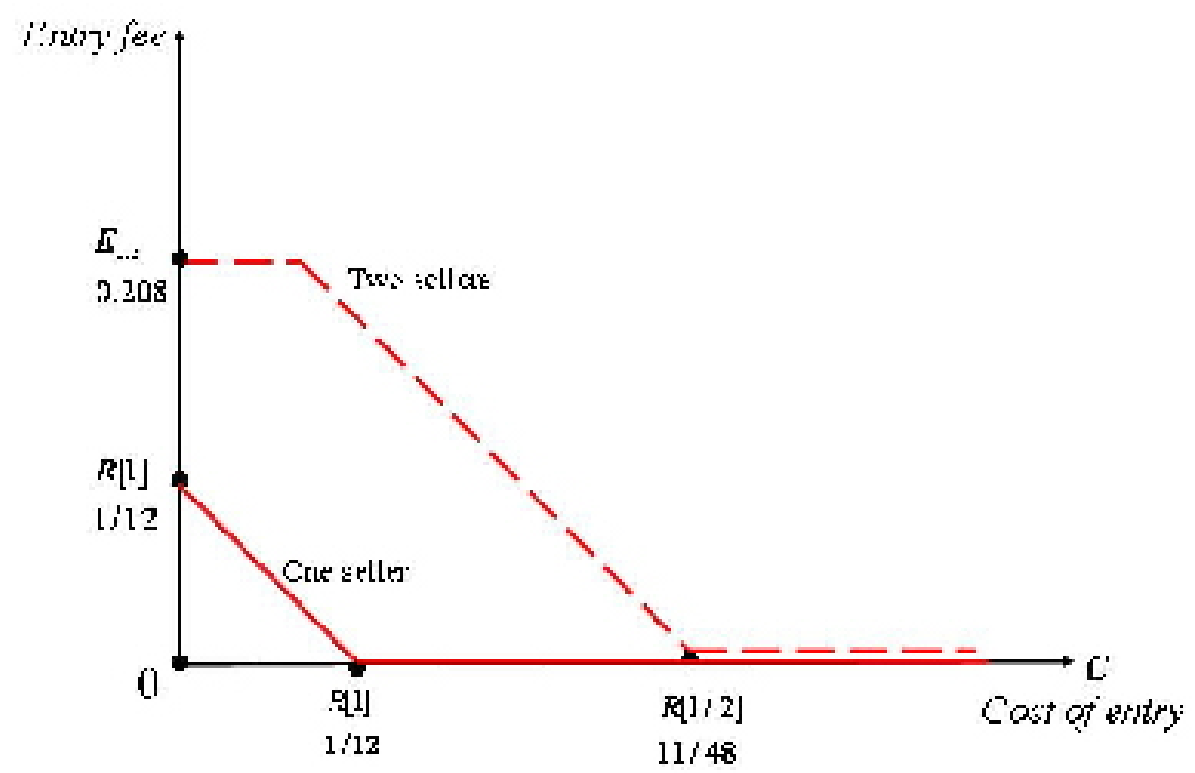

Figure 2: Numerical example: equilibrium entry fees in a market with three buyers, and one seller (the firm line) and two sellers (the dash line), respectively. Buyer values are uniformly distributed. 
result, the buyer competition with the other seller will increase, and the expected payoff of buyers will decrease. The redistribution of buyers will continue until their expected payoffs from visiting the two sellers equalize. That is, raising the entry fee in the case of duopoly will lead to a lower outflow of buyers as compared to the monopoly case. ${ }^{20}$ Because of this, a seller is losing less customers by raising his entry fee in the case of duopoly. This creates an incentive for the sellers to increase their entry fees beyond the entry fee that a monopolist would use as was illustrated in the graph.

\subsection{Buyer and seller surplus}

This model assigns a different role to buyers and sellers. Sellers seem to play a more active role in the market - the role of the mechanism designer - but, as we will see, this role does not seem to translate into a higher surplus. The next theorem establishes how the trade surplus is split between buyers and sellers in large markets.

Theorem 5 Consider a market with $J$ sellers and $k \cdot J$ buyers. Let buyer values be uniformly distributed, and let the cost of entry into the market be zero. As $J \rightarrow \infty$ the expected surplus of a buyer converges to

$$
\frac{1-\frac{(1+k)}{e^{k}}}{k^{2}}
$$

and the expected surplus of a seller converges to

$$
\left(\frac{2+k}{k \cdot e^{k}}+\frac{k-2}{k}\right)
$$

See the Appendix for a proof. When the market is populated by an equal number of buyers and sellers, i.e. when $k=1$, buyers receive a surplus of $\frac{e-1}{e} \approx 0.264$, and sellers obtain $\frac{3-e}{e} \approx 0.104$. The next table gives a more complete picture of buyer and seller surplus for markets of increasing size and equal number of buyers and sellers.

\footnotetext{
${ }^{20}$ When going from the monopoly to the duopoly case, the number of units goes from 1 to 2 . Thus, not only the competition between the sellers, but also the larger total surplus contributes to the result. Another type of comparison would be a monopoly seller with 2 units, and two sellers offering one unit each. In this case the monopoly seller will charge an entry fee that allows him to expropriate the entire surplus from buyers without causing buyers to exit the market. In a duopoly market buyers in general have a positive surplus.
} 


\begin{tabular}{lccccc}
\hline \# sellers & BS & SS & TS & BS/TS (\%) & SS/TS (\%) \\
\hline 2 & 0.1666 & 0.2500 & 0.4166 & 40.0 & 60.0 \\
3 & 0.2226 & 0.1756 & 0.3982 & 55.9 & 44.1 \\
4 & 0.2386 & 0.1513 & 0.3899 & 61.2 & 38.8 \\
5 & 0.2460 & 0.1392 & 0.3852 & 63.9 & 36.1 \\
6 & 0.2502 & 0.1318 & 0.3820 & 65.5 & 34.5 \\
7 & 0.2528 & 0.1271 & 0.3799 & 66.5 & 33.5 \\
8 & 0.2547 & 0.1236 & 0.3783 & 67.3 & 32.7 \\
9 & 0.2561 & 0.1210 & 0.3771 & 67.9 & 32.1 \\
10 & 0.2570 & 0.1192 & 0.3762 & 68.3 & 31.7 \\
100 & 0.2637 & 0.1050 & 0.3687 & 71.5 & 28.5 \\
1000 & 0.2641 & 0.1038 & 0.3679 & 71.8 & 28.2 \\
$J \rightarrow \infty$ & $(e-2) / e$ & $(3-e) / e$ & $1 / e$ & 71.8 & 28.2 \\
\hline
\end{tabular}

Table 3: BS-buyer surplus; SS-seller surplus; TS=SS+BS-total trade surplus; BS/TS (\%)- percentage of total surplus that accrues to buyers; SS/TS (\%)- percentage of total surplus that accrues to sellers. Buyer values are uniformly distributed; cost of entering the market is zero.

As the table illustrates, only in small markets ( 2 buyers $/ 2$ sellers, and 3 buyers $/ 3$ sellers) seller surplus exceeds buyer surplus. When the number of agents increases, the competition among sellers reduces their share of the surplus. It can also be observed that the size of the market exacerbates the coordination problems of buyers, and the total surplus converges down to $1 / e$ as $J \rightarrow \infty$.

\section{Conclusion}

I presented an analytically tractable model of decentralized trading in which sellers compete for buyers by designing transaction mechanisms. Sellers can offer any direct, incentivecompatible and individually rational mechanism. The model allows for any finite number of buyer and sellers, and the interaction among market participants is purely strategic. Upon observing the available mechanisms, buyers symmetrically randomize across sellers.

This assumption generates a pattern of buyer behavior with several attractive features. 
First, this behavior does not presume any sort of coordination among buyers, and assures that buyers are treated symmetrically. Second, buyers distribute stochastically across sellers. Some sellers might on occasions be visited by a very high number of buyers, others by a very low numbers of buyers, and some might even not be visited by buyers at all. This is a feature often observed in reality. Third, the entry probability in each auction varies continuously with the parameters of the mechanisms (e.g. entry fees), and this allows characterization of the equilibrium for markets of various sizes. By allowing for any number of buyers and sellers, the analysis presents a generalization of Levin and Smith's (1994) monopoly model.

The model states comparative statics predictions that are not available in the received models on decentralized trade. First, duopoly sellers charge higher entry fees compared to a monopoly; yet, as the size of the market increases, the equilibrium entry fees decrease, and eventually converge to zero. Second, the increased number of buyers and sellers exacerbates the coordination problem, and increases the probability that items remain unsold (although this probability has a lower bound). Third, in large markets buyers obtain a higher share of the surplus, and this share increases with the size of the market. These insights should guide empirical research on decentralized markets along new lines - ones that account for the endogeneity of the trading institutions and the size of the market.

The model has both positive and normative implications. Taken to real world markets, the model offers a rationale for the frequent use of absolute auctions (auctions with no reserve price or entry fee). These auctions are popular in a variety of markets ranging from real estate and restaurant equipment, to imported goods, and a number of other goods offered online (see, e.g., Engelbrecht-Wiggans 1987, Engelbrecht-Wiggans 1993). Normatively, the model suggests that sellers should not post reserve prices, or charge entry fees when competing with other sellers, especially when the size of the market is large, or buyers incur substantial costs of entering the market.

The analysis has two important limitations. First, it paints a static picture of trade. Once buyers stochastically distribute across sellers, and the transactions take place, there still remain buyers and sellers who were unable to trade. ${ }^{21}$ It can be expected that they might attempt to trade at a later point, and this will have an effect on the transaction mechanisms used by sellers. Allowing for multiple rounds of trading will the next logical step along this line of research. Second, the model does not treat buyers and sellers

\footnotetext{
${ }^{21}$ When the market consists of $J$ sellers and $k \cdot J$ buyers, the probability that a seller will not be visited by customers converges to $e^{-k}$ as $J \rightarrow \infty$.
} 
symmetrically: sellers are shaping the trading institutions, and buyers are responding to sellers' offers. An alternative approach will allow for intermediaries who facilitate the interaction between both market sides by designing trading mechanisms in which both buyers and sellers participate. These issues remain exciting topics for future research.

\section{Appendix}

\section{Proof of Lemma 2:}

The seller chooses $A_{j}$ so as to maximize his expected payoff,

$$
\Pi_{j}\left(A_{j} ; m\right)=\sum_{n=1}^{I} \operatorname{Pr}[n ; m] \cdot n \cdot \int_{0}^{1} Z_{A_{j}}^{(n)}\left(x_{i}\right) d F\left(x_{i}\right),
$$

subject to the constraint that the expected payoff of each bidder equals $R$ :

$$
R=R_{j}^{i}\left(A_{j} ; m\right)=\sum_{n=1}^{I} \operatorname{Pr}[n-1 ; m] \cdot \int_{0}^{1}\left(x_{i} \cdot P_{A_{j}}^{(n)}\left(x_{i}\right)-Z_{A_{j}}^{(n)}\left(x_{i}\right)\right) d F\left(x_{i}\right) .
$$

The right hand side of the expression sums over the expected payoff of a bidder who faces $0,1, \ldots, I-1$ rivals respectively, multiplied by the probabilities for these events. This constraint can be rewritten as a sum of the expected payoffs of all bidders:

$$
I \cdot m \cdot R=\sum_{n=1}^{I} \operatorname{Pr}[n ; m] \cdot n \cdot \int_{0}^{1}\left(x_{i} \cdot P_{A_{j}}^{(n)}\left(x_{i}\right)-Z_{A_{j}}^{(n)}\left(x_{i}\right)\right) d F\left(x_{i}\right) .
$$

Thus, the seller chooses $A_{j}$ to maximize the expression

$$
\sum_{n=1}^{I} \operatorname{Pr}[n ; m] \cdot n \cdot \int_{0}^{1}\left(x_{i} \cdot P_{A_{j}}^{(n)}\left(x_{i}\right)\right) d F\left(x_{i}\right)-I \cdot m \cdot R .
$$

This expression obviously reaches a maximum when

$$
\int_{0}^{1}\left(x_{i} \cdot P_{A_{j}}^{(n)}\left(x_{i}\right)\right) d F\left(x_{i}\right)
$$

is maximized for every $n=1,2, \ldots, I$. Because $x_{-i} \in[0,1]^{n-1}$ we have

$$
P_{A_{j}}^{(n)}\left(x_{i}\right)=\int_{[0,1]^{n-1}} p_{i}\left(x_{i}, x_{-i}\right) d F\left(x_{-i}\right)
$$


and since I consider only anonymous mechanisms (see condition $(A N)$ ), I obtain

$$
\int_{0}^{1}\left(x_{i} \cdot P_{j}^{(n)}\left(x_{i}\right)\right) d F\left(x_{i}\right)=\frac{1}{n} \cdot \int_{[0,1]^{n}}\left(x_{i} \cdot p_{i}(x)\right) d F(x) .
$$

The expression reaches a maximum when, for every participating bidder $i$, probability $p_{i}(x)$ is chosen so that

$$
p_{i}(x)= \begin{cases}1 & \text { if } x_{i} \text { is the highest valuation } \\ 0 & \text { otherwise }\end{cases}
$$

\section{Proof of Theorem 2:}

Assume by contradiction $A^{C}$ is not an equilibrium in the game with a strategy set $\Omega$. Hence, there exists a deviation mechanism $\tilde{A}_{j}$ with entry fees $\tilde{C}_{1}, \tilde{C}_{2}, \ldots, \tilde{C}_{I}$ which brings seller $j$ a higher expected payoff: $\Pi_{j}\left(\tilde{A}_{j} ; \tilde{m}\right)>\Pi_{j}\left(A_{j}^{C} ; \tilde{m}\right)$, where $\tilde{m}$ is the probability that bidders enter the mechanism of seller $j$. The expected payoff of bidder $i$ is

$$
R_{j}^{i}\left(\tilde{A}_{j} ; \tilde{m}\right)=\sum_{n=1}^{I} \operatorname{Pr}[n-1 ; \tilde{m}] \cdot B_{n}-\sum_{n=1}^{I} \operatorname{Pr}[n-1 ; \tilde{m}] \cdot \tilde{C}_{n}
$$

and of seller $j$ is

$$
\Pi_{j}\left(\tilde{A}_{j} ; \tilde{m}\right)=\sum_{n=1}^{I} \operatorname{Pr}[n ; \tilde{m}] \cdot S_{n}+\sum_{n=1}^{I} \operatorname{Pr}[n ; \tilde{m}] \cdot n \cdot \tilde{C}_{n}
$$

Let us construct now a mechanism $A_{j}^{\tilde{C}}$ with a constant entry fee

$$
\tilde{C}=\sum_{n=1}^{I} \operatorname{Pr}[n-1 ; \tilde{m}] \cdot \tilde{C}_{n} .
$$

The equilibrium assumption (B2) guarantees that buyers will continue to visit seller $j$ with a probability of $\tilde{m}$ because their expected payoff has not changed. The payoff of the seller is now

$$
\Pi_{j}\left(A_{j}^{\tilde{C}} ; \tilde{m}\right)=\sum_{n=1}^{I} \operatorname{Pr}[n ; \tilde{m}] \cdot S_{n}+\sum_{n=1}^{I} \operatorname{Pr}[n ; \tilde{m}] \cdot n \cdot \tilde{C}=\sum_{n=1}^{I} \operatorname{Pr}[n ; \tilde{m}] \cdot S_{n}+I \cdot \tilde{m} \cdot \tilde{C}
$$


From equation $(C E)$ follows

$$
\sum_{n=1}^{I} \operatorname{Pr}[n ; \tilde{m}] \cdot n \cdot \tilde{C}=\sum_{n=1}^{I} \operatorname{Pr}[n ; \tilde{m}] \cdot n \cdot \tilde{C}_{n}
$$

because

$$
I \cdot \tilde{m} \cdot \tilde{C}=I \cdot \tilde{m} \cdot\left(\sum_{n=1}^{I} \operatorname{Pr}[n-1 ; \tilde{m}] \cdot \tilde{C}_{n}\right)=\sum_{n=1}^{I} \operatorname{Pr}[n ; \tilde{m}] \cdot n \cdot \tilde{C} .
$$

As each bidder pays the same expected fee, the expected fees that the seller obtains in the two mechanisms are equal. It follows

$$
\Pi_{j}\left(A_{j}^{\tilde{C}} ; \tilde{m}\right)=\Pi_{j}\left(\tilde{A}_{j} ; \tilde{m}\right)>\Pi_{j}\left(A_{j}^{C} ; \tilde{m}\right) .
$$

The mechanism $A_{j}^{\tilde{C}} \in \Omega_{e}$ brings seller $j$ a higher expected payoff than the equilibrium mechanism $A_{j}^{C}$, and this yields the desired contradiction.

\section{Proof of Lemma 4:}

Let $J S\left(m_{i}, m\right)$ denote the joint surplus of the seller and all bidders, if bidder $i$ participates with a probability of $m_{i}$ and all other bidders with a probability of $m$. The expected gain of bidder $i$ is $m_{i} \cdot R[m]$, and from Lemma 3 follows $J S\left(m_{i}, m\right)=m_{i} \cdot R[m]$. The identities

$$
\frac{d}{d m}(J S(m))=I \cdot \frac{\partial}{\partial m_{i}}\left(\left.J S\left(m_{i}, m\right)\right|_{m_{i}=m}=I \cdot R[m]\right.
$$

yield the desired result.

\section{Proof of Theorem 4:}

The entry fee is given by $E_{J, k J}=\left.\frac{1}{J} \cdot \frac{d}{d m}\left(R\left[\frac{1-m}{J-1}\right]\right)\right|_{m=1 / J}$. Observe that $\frac{d}{d m}\left(R\left[\frac{1-m}{J-1}\right]\right)=$ $-\frac{1}{J-1} \cdot \frac{d R[m]}{d m}$, and hence

$$
E_{J, k J}=-\frac{1}{J \cdot(J-1)} \cdot \frac{d R[m]}{d m} .
$$

Let us denote by $R\left[m_{i}, m\right]$ the expected payoff of a bidder who participates in an auction with no entry fee or a reserve price, given that all other bidders participate with a 
probability of $m$ except for one, who enters the auction with a probability of $m_{i}$. Then

$$
\begin{aligned}
& \frac{d R[m]}{d m}\left|{ }_{m=1 / J}=I \cdot \frac{\partial}{\partial m_{i}} R\left[m_{i}, m\right]\right|_{m_{i}=m=1 / J} \Leftrightarrow \\
& \frac{d R[m]}{d m} \mid{ }_{m=1 / J}=k J \cdot \frac{\partial}{\partial m_{i}}\left(m_{i} \cdot R[1, m]+\left(1-m_{i}\right) \cdot R[0, m]\right) \Leftrightarrow \\
& \frac{d R[m]}{d m} \mid{ }_{m=1 / J}=k J \cdot(R[1, m]-R[0, m]) .
\end{aligned}
$$

Hence

$$
E_{J, k J}=\frac{k}{J-1} \cdot(R[0, m]-R[1, m]) .
$$

Recall that $R[m]=\sum_{n=1}^{I} \operatorname{Pr}[n-1 ; m] \cdot B_{n}$ and observe that

$$
R[0, m]-R[1, m]=\sum_{n=1}^{k J} \operatorname{Pr}[n-1 ; m] \cdot\left(B_{n-1}-B_{n}\right)<\sum_{n=1}^{k J} \operatorname{Pr}[n-1 ; m] \cdot B_{0}=B_{0}
$$

The claim follows.

\section{Proof of Theorem 5:}

The seller surplus is given by:

$$
\begin{aligned}
\lim _{J \rightarrow \infty} \sum_{n=1}^{k \cdot J} \operatorname{Pr}[n ; 1 / J] \cdot S_{n} & =\lim _{J \rightarrow \infty} \sum_{n=1}^{k \cdot J} \operatorname{Pr}[n ; 1 / J] \cdot \frac{n-1}{n+1} \\
& =\lim _{J \rightarrow \infty} \sum_{n=1}^{k \cdot J} \operatorname{Pr}[n ; 1 / J] \cdot\left(1-\frac{2}{n+1}\right) \\
& =\lim _{J \rightarrow \infty} \sum_{n=1}^{k \cdot J} \operatorname{Pr}[n ; 1 / J]-2 \cdot \lim _{J \rightarrow \infty} \sum_{n=1}^{k \cdot J} \operatorname{Pr}[n ; 1 / J]\left(\frac{1}{n+1}\right) \\
& =1-\lim _{J \rightarrow \infty}\left(1-\frac{1}{J}\right)^{k J}-2 \cdot \lim _{J \rightarrow \infty} \sum_{n=1}^{k \cdot J} \operatorname{Pr}[n ; 1 / J]\left(\frac{1}{n+1}\right) \\
& =\left(1-\frac{1}{e^{k}}\right)-2 \frac{\left(1-\frac{k+1}{e^{k}}\right)}{k}=\frac{2+k}{k \cdot e^{k}}+\frac{k-2}{k} .
\end{aligned}
$$

The result presented in the last line is established by using the identity

$$
\left(\frac{n}{k}\right)=\frac{n}{k} \cdot\left(\frac{n-1}{k-1}\right)
$$


and observing that

$$
\begin{aligned}
\lim _{J \rightarrow \infty} \sum_{n=1}^{k \cdot J} \operatorname{Pr}[n ; 1 / J]\left(\frac{1}{n+1}\right) & =\lim _{J \rightarrow \infty} \frac{1}{k \cdot J+1} \sum_{n=1}^{k \cdot J}\left(\frac{k \cdot J+1}{n+1}\right)\left(\frac{1}{J}\right)^{n}\left(1-\frac{1}{J}\right)^{k \cdot J-n} \\
& =\lim _{J \rightarrow \infty} \frac{1}{k \cdot J+1} \sum_{n=2}^{k \cdot J+1}\left(\frac{k \cdot J+1}{n}\right)\left(\frac{1}{J}\right)^{n}\left(1-\frac{1}{J}\right)^{k \cdot J-n+1} \\
& =\lim _{J \rightarrow \infty} \frac{J}{(k \cdot J+1)} \sum_{n=2}^{k \cdot J+1}\left(\frac{k \cdot J+1}{n}\right)\left(\frac{1}{J}\right)^{n-1}\left(1-\frac{1}{J}\right)^{k \cdot J-n+1} \\
& =\lim _{J \rightarrow \infty} \frac{J}{(k \cdot J+1)}\left(-(k \cdot J+1)\left(\frac{1}{J}\right)\left(1-\frac{1}{J}\right)^{k \cdot J}\right) \\
& =\lim _{J \rightarrow \infty} \frac{1}{\left(k+\frac{1}{J}\right)}\left(\begin{array}{c}
1-\left(1-\frac{1}{J}\right)^{k \cdot J+1} \\
-\left(k+\frac{1}{J}\right)\left(1-\frac{1}{J}\right)^{k \cdot J}
\end{array}\right) \\
& =\lim _{J \rightarrow \infty} \frac{1}{\left(k+\frac{1}{J}\right)}-\lim _{J \rightarrow \infty}\left(\frac{\left(1-\frac{1}{J}\right)^{k \cdot J+1}}{\left(k+\frac{1}{J}\right)}-\left(1-\frac{1}{J}\right)^{k \cdot J}\right) \\
& =\frac{1}{k}-\frac{e^{-k}-e^{-k}=\frac{\left(1-\frac{k+1}{e^{k}}\right)}{k} .}{k}
\end{aligned}
$$

In equilibrium buyers earn $R[1 / J]$ minus the entry fee, which converges to zero as $J \rightarrow \infty$. Thus, the buyer surplus is given by ${ }^{22}$

$$
\begin{aligned}
\lim _{J \rightarrow \infty} \sum_{n=1}^{k \cdot J} \operatorname{Pr}[n-1 ; 1 / J] \cdot B_{n} & =\lim _{J \rightarrow \infty} \sum_{n=1}^{k \cdot J} \operatorname{Pr}[n-1 ; 1 / J] \cdot \frac{1}{n(n+1)} \\
& =\lim _{J \rightarrow \infty} \sum_{n=1}^{k \cdot J} \operatorname{Pr}[n-1 ; 1 / J] \cdot\left(\frac{1}{n}-\frac{1}{n+1}\right) \\
& =\lim _{J \rightarrow \infty} \sum_{n=1}^{k \cdot J} \operatorname{Pr}[n-1 ; 1 / J] \cdot \frac{1}{n}-\lim _{J \rightarrow \infty} \sum_{n=1}^{k \cdot J} \operatorname{Pr}[n-1 ; 1 / J] \cdot \frac{1}{n+1} \\
& =\frac{k-\frac{k}{e^{k}}}{k^{2}}-\frac{k-1+\frac{1}{e^{k}}}{k^{2}}=\frac{1-\frac{(1+k)}{e^{k}}}{k^{2}} .
\end{aligned}
$$

\footnotetext{
${ }^{22}$ The expression in the last line is similarly obtained by changing the boundaries of the sum and using identity (I).
} 


\section{References}

Burguet, R., and J. Sakovics (1999): "Imperfect Competition in Auction Designs," International Economic Review, 40, 231-247.

Chakraborty, I., and G. Kosmopoulou (2001): "Auctions with Endogenous Entry," Economics Letters, 72, 195-200.

Engelbrecht-Wiggans, R. (1987): "On Optimal Reservation Prices in Auctions," Management Science, 33, 763-770.

239.

Hernando-Veciana, A. (2005): "Competition among Auctioneers in Large Markets," Journal of Economic Theory, 121, 107-127.

Levin, D., And J. L. Smith (1994): "Equilibrium in Auctions with Entry," American Economic Review, 84, 585-599.

Marmer, V., A. Shneyerov, and P. Xu (2007): "What Model of Entry in First-Price Auctions? A Nonparametric Approach," Working paper, University of British Columbia.

McAfee, R. P. (1993): "Mechanism Design by Competing Sellers," Econometrica, 61, $1281-1312$.

McAfee, R. P., And J. McMillan (1987a): "Auctions and Bidding," Journal of Economic Literature, 30, 699-738.

(1987b): “Auctions with Entry," Economics Letters, 23, 343-347.

Moldovanu, B., A. Sella, and X. Shi (2008): "Competing Auctions with Endogenous Quantities," Journal of Economic Theory, forthcoming.

Myerson, R. (1997): Game Theory: Analysis of Conflict. Harvard University Press, Cambridge, Massachusetts.

Peters, M. (1994): "Equilibrium Mechanisms in a Decentralized Market," Journal of Economic Theory, 64, 390-423. 
(1997): "A Competitive Distribution of Auctions," Review of Economic Studies, 64, 97-123.

Peters, M., and S. Severinov (1997): "Competition among Sellers Who Offer Auctions Instead of Prices," Journal of Economic Theory, 75, 141-179.

Riley, J. G., and W. F. Samuelson (1981): "Optimal Auctions," American Economic Review, 71, 381-392.

Samuelson, W. (1985): "Competitive Bidding with Entry Costs," Economics Letters, 17, $53-57$. 